\title{
OPTIMIZATION OF SAMPLE PREPARATION FOR THE DETERMINATION OF HPA'S VISION IN A GEOMETRIC TECHNIQUE USING MOLECULAR FLUORESCENCE
}

\author{
BARBOSA, Andréa Francisca Fernandes $\left({ }^{*}\right)^{1}$; MOURA, Dayanne Chianca ${ }^{2}$; HUSSEIN, \\ Fabiana Roberta Gonsalves da Silva3; MELO, Jailson Vieira4; SILVA, Djalma Ribeiro ${ }^{5}$. \\ ${ }^{1}$ Universidade Potiguar, Unidade de Engenharia, Av. Nascimento de Castro,1597, CEP 59056-450, Natal-RN, \\ Brasil (fone: 84 40091414); \\ 2,3,4,5 Universidade Federal do Rio Grande do Norte, Instituto de Química, Campus Universitário, Lagoa Nova, \\ CEP 59078- 970, Natal-RN, Brasil \\ ${ }^{*}$ Autor Correspondente \\ *e-mail: andreabarbosa@unp.br
}

Received 24 July 2012; received in revised form 23 August 2012; accepted 27 August 2012

\section{RESUMO}

A preparação de amostra é considerada uma etapa primordial no processo de análise. A complexidade da matriz e a forma como os compostos estão ligados é uma das principais dificuldades na extração. Assim um sistema de solvente aliado a um método de extração, ambos precisam ser eficientes frente a esses percalços. Neste trabalho utilizou-se o planejamento fatorial 23 para determinar as condições ótimas para a preparação da amostra. A análise das amostras foram realizadas em um Espectrofotômetro de fluorescência molecular. Os fatores escolhidos para investigar a influência no sistema de extração foram: tempo de utilização do ultrassom, o tipo de solvente (hexano ou diclorometano) e proporção solvente/amostra (1:1 e 1:3). O melhor resultado foi obtido com a utilização do diclorometano, com o tempo de 10 min de ultrassom e a proporção solvente/amostra $1: 3$.

Palavras-chave: Visão Geométrica, Preparação de Amostra, Fluorescência Molecular, Planejamento Fatorial $2^{3}$.

\section{ABSTRACT}

The sample preparation is considered an essential step in the analysis process. The complexity of the matrix and how the compounds are linked is one of the main difficulties in the extraction. Thus a solvent system combined with an extraction method, both need to be effective against such mishaps. In this work we used the 23 factorial design to determine the optimum conditions for sample preparation. The analysis of the samples were performed in a molecular fluorescence spectrophotometer. The factors selected to investigate the influence on the extraction system were: time of use of ultrasound, the type of solvent (hexane or dichloromethane) and ratio solvent / sample (1:1 and 1:3). The best result was obtained with using the dichloromethane in time of 10 min of ultrasound and the ratio solvent / sample 1:3.

Keywords: geometric vision, sample preparation, molecular fluorescence, factorial design. 


\section{INTRODUÇÃO}

Qualquer amostra, sendo ela orgânica ou inorgânica, necessita passar pela etapa de preparação antes de ser analisada. A preparação da amostra exige uma criteriosa extração pois essa etapa é determinante para a análise da mesma. O sistema de extração ideal deve permitir que todos os compostos de interesse presentes na amostra sejam totalmente extráidos para a fase orgânica para que se tenha um resultado confiável, evitandose assim, os falsos positivos, embora a incerteza esteja presente. A prepararação da amostra requer tempo para extrair os analitos de interesse. A água mesmo sendo um solvente universal não é adequado para análise, pois a mesma mascara os resultados, não havendo absorção dos compostos neste meio por fluorescência molecular. A literatura apresenta várias metodologias para extração de orgânicos em amostras aquosas, entretanto essas metodologias não justificam a escolha do solvente no processo de extração. Por isso, foi utilizado um planejamento fatorial $2^{3}$ (Barros, 2003; Rodrigues, 2005) com interpretação num cubo geométrico 3D para analisar os resultados e determinar a combinação adequada. $A$ visão estatística de um planejamento fatorial $2^{3}$ fornece a condição ótima para preparação da amostra e posterior análise (Leite, 2005). Os parâmetros utilizados neste planejamento foram: tipo de solvente (diclorometano ou hexano); proporção solvente/amostra; e a influência do ultrassom. A resposta foi obtida através de um Espectrofotômetro de Fluorescência Molecular (Queiroz et. al. 2005). A integração da área dos picos dos espectros foram calculadas e associadas à concentração dos analitos em cada amostra do planejamento fatorial que resultou em 8 experimentos. Essa área foi calculada através da relação comprimento de onda $(\mathrm{nm})$ versus intensidade do pico em cada uma das 8 amostras obtidas.

A amostra sintética preparada continha os 16 HPAs (Hidrocarbonetos Policíclicos Aromáticos) prioritários pela USEPA (United States Enviromental Protection Agency) (Bohm et al. 2002) como forma de observar a eficiência de extração daquela amostra.

Os HPAs são compostos orgânicos provenientes do petróleo que apresentam uma estrutura cíclica variando de dois a seis anéis.
Eles têm recebido mais atenção desde que foram encontrados no solo pela primeira vez (Blumer, 1975, p. 53-55; Sissino et. al. 2003; Artiola, 2004; USEPA, 1999). Os HPAs recebem uma atenção considerável devido às propriedades mutagênicas, teratogênicas e carcinogênicas de alguns deles (Costa, 2001; Yu et. al. 2004; Arbex, 2001).

Neste trabalho os HPAs foram analisados pela técnica de Fluorescência Molecular no ultravioleta (Queiroz et. al. 2005; Atkins, 2002), que tem sido amplamente empregada, devido à rapidez e facilidade analítica, ao custo relativamente baixo e a alta sensibilidade. Entretanto, esse método apresenta limitação capaz de diferenciar hidrocarbonetos diferentes, (Neves, 2006, p.10 - 15). Os principais objetivos foram: 1) investigar a influência de três fatores que são considerados determinantes no processo de extração: tipo de solvente (Cienfuegos, 2002); proporção solvente/amostra; utilização do ultrassom (Kummrow, 2004) - utilizando a técnica analítica de fluorescência molecular, 2) utilizar a interpretação geométrica para determinar a condição ótima de extração líquido-líquido dos HPAs (Giessing, 2003).

\section{DESENVOLVIMENTO}

Neste trabalho foi utilizado uma amostra sintética de água deionizada contaminada com os 16 HPAs prioritários (Boehm et al. 2002). Utilizou-se um planejamento fatorial $2^{3}$ para verificar a melhor combinação do sistema de extração da amostra sintética (Brito, 2003). A combinação dos três fatores (tipo de solvente, proporção solvente/amostra, uso do ultrassom) em dois níveis (mínimo e máximo) resultou em 8 ensaios para a extração líquido-líquido das amostras sintéticas de água como mostra a Tabela 1.

A extração das amostras dos 8 experimentos foram realizadas aleatoriamente (através de sorteio) a fim de se diminuir a propagação de erros. Depois de submetidas às combinações do planejamento fatorial, os extratos orgânicos foram levados ao espectrofotômetro de fluorescência para serem analisados. Os espectros obtidos do espectrofluorímetro foram plotados no software 
Origin Pro 7.5 para posterior cálculo da área (Barbosa, 2008). As 8 combinações do planejamento fatorial $2^{3}$ estão apresentadas na Tabela 2 com seus respectivos níveis mínimo $(-)$ e máximo $(+)$ de cada experimento.

\section{RESULTADOS E DISCUSSÃO:}

Os resultados em termos de concentração, obtidos dos espectros de fluorescência molecular (Castillo, 2004) através da relação comprimento de onda $(\mathrm{nm})$ versus intensidade de cada solução sintética obtida, foram colocados em um cubo geométrico 3D para se determinar o melhor sistema de extração da amostra sintética preparada. E por fim verificar qual o tipo de influência de cada fator investigado. A interpretação do cubo geométrico nos permitiu identificar como os fatores influenciam no sistema de extração da amostra.

Cada um dos 8 vértices do cubo corresponde às respostas (concentrações dos extratos orgânicos nas 8 combinações do planejamento $2^{3}$ ) que pode ser verificado na representação da Figura 1.

Observando o cubo da Figura 1, podese verificar que 0 uso do solvente diclorometano (nível máximo $(+)$ ) influencia positivamente na extração, uma vez que apresenta uma elevação na concentração em todos os ensaios nos quais ele é utilizado. Contudo, consegue-se uma otimização do processo quando se utiliza o diclorometano juntamente com a proporção solvente/amostra 1:3 (também nível máximo $(+)$ ). 0 fato do diclorometano possuir um poder de extração superior ao hexano se deve ao valor do seu índice polar. O hexano apresenta um índice polar bastante baixo em comparação ao diclorometano. Além disso, o hexano apresenta uma toxidade maior por ser um solvente bastante volátil. Analisando o cubo geométrico quando comparamos os vértices: $1 \mathrm{com} \mathrm{2;3}$ com 4; 5 com 6; 7 com 8, verificamos um aumento nas respostas. Isso evidencia positivamente a influência do uso diclorometano. Para analisar a proporção solvente/amostra comparamos os vértices 1 com 5; 2 com 6; 3 com 7; 4 com 8. Percebe-se que as melhores respostas são obtidas através da proporção 1:3 solvente/amostra. E por fim analisamos a influência do uso do ultrassom comparando-se as respostas dos vértices 1 com 3; 2 com 4; 5 com 7; 6 com 8. Verifica-se também que o uso do ultrassom aumenta significativamente as respostas.

\section{CONCLUSÕES:}

A otimização do processo de extração de HPAs de uma amostra aquosa exige alguns requisitos que devem ser levados em consideração para se ter um resultado confiável, evitando-se assim os falsos positivos. Por isso, o trabalho acima descrito foi realizado com a ajuda de um planejamento fatorial que permitiu verificar a influência dos fatores combinados em seus dois níveis, mínimo e máximo. Portanto, a combinação do planejamento fatorial $2^{3}$ utilizando o solvente diclorometano na proporção 1:3 solvente/amostra com agitação no ultrassom forneceu uma melhor e satisfatória resposta, indicando assim, uma eficiente extração dos compostos de interesse. A utilização da interpretação geométrica do cubo revelou que:

a) Pôde-se observar uma diferença entre os dois tipos de solventes utilizados, entretanto, não pode-se descartar a possibilidade do uso do hexano que também apresentou bons valores na extração dos HPAs;

(b) A proporção 1:3 mostrou em todas as combinações valores representativos quando na sua interação entre o diclorometano, e;

(c) A utilização do ultrassom indiscutivelmente favorece a recuperação dos HPAs.

\section{AGRADECIMENTOS:}

Ao laboratório de pesquisa NUPRAR da UFRN que contribuiu para realização das análises.

\section{REFERÊNCIAS:}

1. ARBEX, M. A., Avaliação dos Efeitos do Material Particulado Proveniente da Queima da Plantação da Queima de cana-de-açúcar sobre a Morbidade Respiratória na População de Araraguara - SP. 2001. 188 f. Tese (Doutorado em Medicina) - Faculdade de Medicina da Universidade de São Paulo, São Paulo, 2001. p. 46. 
2. ARTIOLA, J. F. Environmental Monitoring and Characterization. USA: Elsevier, 2004, p.284. ISBN: 0120644770.

3. ATKINS, Peter; PAULA, Julio de. Físico-Química.7. ed. Rio de Janeiro: Ed. LTD, 2002. vol. 2, p. 268-269.

4. BARBOSA, A. F. F., Otimização de metodologias para determinação de hidrocarbonetos policíclicos aromáticos:aplicação em água do Rio Potengi, 2008, 162f, tese (Doutorado em química) - Universidade Federal do Rio Grande do Norte. Centro de Ciências Exatas e da Terra. Programa de Pós-Graduação em Química.

5. BARROS NETO, Benício de; SCARMINIO, leda Spacino; BRUNS, Roy Edward. Como fazer experimentos: pesquisa e desenvolvimento na indústria. 2. ed. Campinas - SP: Ed. UNICAMP, 2003.p. 95103.

6. BLUMER, M.; YONUMBLOOD, W. W. Polycyclic Aromatic Hydrocarbons in Soils and Recent Sediment, Science, v. 188, n. 4183, p. 53-55, 1975. Disponível em: https://www.sciencemag.org/cgi/content/abstract/188/4183/53.

7. BOEHM, P.D.; LORETI, C. P.; ROSENSTEIN A. B.; RURY, P. M., A Guide to Polycyclic. Aromatic Hydrocarbons for the NonSpecialist, American Petroleum Institute, fev. 2002. p. 2-4.

8. CIENFUEGOS, Freddy. Tabelas Químicas. Rio de Janeiro: Interciência, 2002. p. 92.

9. COSTA, A. F., "Avaliação da Contaminação Humana por Hidrocarbonetos Policíclico

Aromáticos (Hpas): 1-Hidróxido Pireno Urinário". 2001. 81f. Dissertação (Mestrado em saúde pública) - Escola Nacional de Saúde Pública, Rio de Janeiro, 2001. p. 2-10.

10. BRITO, Natilene Mesquita; et. al. Validação de Métodos Analíticos: Estratégia e Discussão,Pesticidas: Revista Ecotoxicologia e Meio Ambiente, v. 13, jan./dez. 2003. p. 129-146. Disponível em: http://ojs.c3sl.ufpr.br/ojs2/in- dex.php/pesticidas/issue/view/333. Acesso em: 21/05/2007.

11. CASTILLO, A. S.; et. al. Heavy Atom Induced Room Temperature Phosphorescence: a Tool for the Analytical Characterization of Polycyclic Aromatic Hydrocarbons, Analytica Chimica Acta, v. 516, n. 1-2, p. 213-220, 19 jul. 2004. Disponível em: http://www.sciencedirect.com/science_ob=ArticleURL\&_udi=B6TF44CF5DV7-

$5 \&$ user $=687335 \&$ coverDate $=07 \% 2 \mathrm{~F} 19 \% 2 \mathrm{~F} 2$ $004 \&$ alid $=970373623 \&$ rdoc $=13 \&$ fmt $=$ high \&

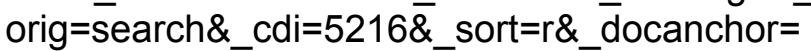
\&view $=c \&$ ct $=15 \& \_a c c t=\bar{C} 000037878 \&$ version $=1 \&$ urlVersion $=0 \&$ userid $=687335 \& \mathrm{md} 5=$ 5ae2cf72849b83961e12036b7f5090c.

12. Fernandes, P. R. N.; Pinheiro, L. S.; Nascimento, R. F. do; Soares, S. de A.; Avaliação da presença de hidrocarbonetos policíclicos aromáticos (hpas) em ligante asfáltico brasileiro. $4^{\circ}$ PDPETRO, Campinas, SP, 2007. Disponível http://www.portalabpg.org.br/PDPetro/4/resumos/4PDPETRO_6_2_0392-1.pdf. Acessado em: 07/08/2012.

13. GIESSING, A., M. B.; MAYER, L. M.; FORBES, T. L., Synchronous Fluorescence Spectrometry of 1-Hydroxypyrene: a Rapid Screening Method for Identification of PAH Exposure in Tissue from Marine Polychaetes, Marine Environmental Research, v. 56, n.5, p. 599-615, dez. 2003.

14. KUMMROW, F. et. al. Uso da técnica de Blue rayon in situ para extração/concentração de

compostos orgânicos policíclicos genotóxicos em ambientes aquáticos. In: IV CONGRESSO BRASILEIRO DE PESQUISAS AMBIENTAIS E SAÚDE - CBPAS 2004, Santos. Anais do CBPAS, Santos: Universidade Católica de Santos, 2004, v. 39, p. 39.

15. LEITE, F., Validação em Análise Química. 4. ed. Campinas - SP: Ed. Átomo, 2005.

16. NEVES, Roberta Lyrio Santos. Avaliação da Contaminação de Óleo no Ambiente Estuarino da Baía De Guanabara (RJ) pela Determinação Fluorimétrica de Hidrocarbo- 
netos Policíclicos Aromáticos (HPA's) na Bílis de Peixes Mugil Liza. 2006. 120 f. Dissertação (Mestrado em Química) - Pontifícia Universidade Católica do Rio de Janeiro. Programa de Pós- Graduação em Química, Rio de Janeiro, 2006.

17. QUEIRÓS, Y. G. C.; CLARISSE, M. D.; OLIVEIRA, R. S.; REIS, B. D.; TRAVALLONI, A. M.L. E LUCAS, E. F.; Determinação e correlação do teor total de óleos e graxas por meio de diferentes técnicas espectroscópicas e gravimétrica, $3^{\circ}$ Congresso Brasileiro de P\&D em Petróleo e Gás, 2005.

18. RODRIGUES, Maria Isabel; IEMMA, Antonio Francisco. Planejamento de Experimentos e Otimização de Processos. 1. ed. Campinas - SP: Ed. Casa do Pão, 2005.p. 4-6.

19. SISINNO, C. L. S.; NETTO, A. D. P.; Rego, E. C. P.; LIMA, G. dos S. V.; Hidrocarbonetos policíclicos aromáticos em resíduos sólidos industriais: uma avaliação preliminar do risco potencial de contaminação ambiental e humana em áreas de disposição de resíduos, Cad. Saúde Pública, Rio de Janeiro, 19(2), 2003. p. 671676.

20. YU, Ming-Ho. Environmental Toxicology: Biological and Health Effects of Pollutants, 2. ed.New Work: Editora CRC Press, 2004. p. 36-41.

21. USEPA - UNITED STATE ENVIROMENT PROTECT AMBIENTAL, Compendium of Methods for Toxic Organic Air Pollutants, Second Edition, 625/R-96/010b, January 1999, p 13. 
Tabela 1. Fatores utilizados na análise com os respectivos valores dos níveis mínimo (-) e máximo $(+)$.

\begin{tabular}{c|c|c}
\hline \multirow{2}{*}{ Fatores } & \multicolumn{2}{|c}{ Níveis } \\
\cline { 2 - 3 } & $(-1)$ & $(+1)$ \\
\hline Solvente & Hexano & Diclorometano \\
\hline $\begin{array}{c}\text { Proporção } \\
\text { Solvente/Amostra }\end{array}$ & $1: 1$ & $1: 3$ \\
\hline Ultrassom & Sem & Com \\
\hline
\end{tabular}

Tabela 2. Matriz de planejamento dos ensaios.

\begin{tabular}{c|c|c|c}
\hline Ensaio & Solvente & $\begin{array}{c}\text { Proporção } \\
\text { Solvente/Amostra }\end{array}$ & Ultrassom \\
\hline 1 & - & - & - \\
\hline 2 & + & - & - \\
\hline 3 & - & + & - \\
\hline 4 & + & + & - \\
\hline 5 & - & - & + \\
\hline 6 & + & - & + \\
\hline 7 & - & + & + \\
\hline 8 & + & + & + \\
\hline
\end{tabular}

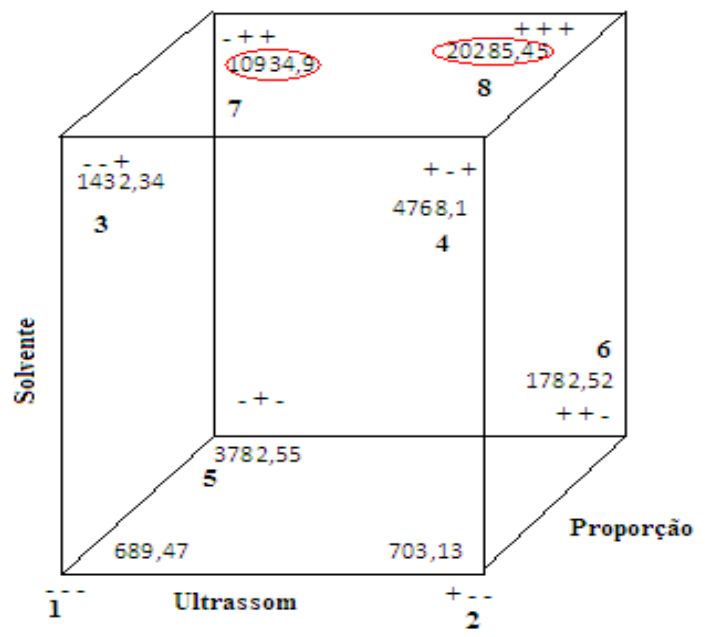

Figura 1. Representação do cubo com as respostas em cada um dos oito vértices referentes aos oito ensaios do planejamento fatorial $2^{3}$.

PERIÓDICO TCHÊ QUÍMICA • www.periodico.tchequimica.com • Vol. 9 N. 18 •ISSN 1806-0374 (impresso) • ISSN 1806-9827 (CD-ROM) • ISSN 2179-0302 (meio eletrônico) 